\title{
A High Sensitivity Biosensor for Cellular Metabolism Monitoring Based on Improved Light-addressable Potentiometric Sensor and Constant Voltage Detection
}

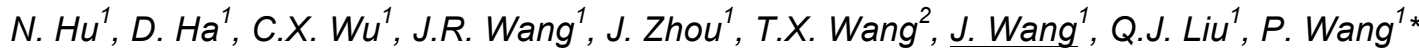 \\ ${ }^{1}$ Biosensor National Special Laboratory, Key Laboratory for Biomedical Engineering of Education \\ Ministry, Department of Biomedical Engineering, Zhejiang University, Hangzhou, P. R. China, 310027 \\ ${ }^{2}$ ACEA Bioscience Inc., West Lake Technology and Economy Park, Hangzhou, 310030, P. R. China \\ ${ }^{+}$Corresponding author E-mail: cnpwang@zju.edu.cn Tel: +86 57187952832
}

\begin{abstract}
:
A high sensitivity biosensor based on light-addressable potentiometric sensor (LAPS) was established for monitoring the cellular metabolism. Constant voltage detection mode was introduced to replace the conventional constant current mode. Moreover, the sensitivity of LAPS surface potential was improved by reducing the thickness of the semiconductor substrate. By these means, the sensitivity of cellular metabolism monitoring was $1104 \mathrm{nA} / \mathrm{pH}$ in the most of extracellular microenvironment (pH6-pH8). Cellular metabolism experiments were carried out to determine the sensor performance. The result showed that characteristics of LAPS have a better performance to sensor the change of cellular metabolism rapidly and sensitively in real time detection. By the improved LAPS and the novel detection mode, the high sensitivity biosensor is promising to improve to study the mechanism of cellular metabolism.
\end{abstract}

Key words: high sensitivity biosensor, light-addressable potentiometric sensor (LAPS), constant voltage detection, cellular metabolism

\section{Introduction}

Cellular metabolism is a common biological mechanism involved in many significant physiological processes. For a living cell, thousands of biological reactions happen which relate to each other in a complicated network in vivo. Most of the biological reactions depend on the energy, and then the cascade reactions are tightly coupled. Finally, the metabolic processes provide energy ATP for the normal physiological activities of cells. (See Fig.1(A)) The cellular metabolism begins with the uptake of oxygen, glucose and other nutrients, then degrades these nutrients to generate the energy ATP and finally secretes the acidic products. In aerobic condition, via the glycolysis, citric acid cycle and oxidative phosphorylation, glucose is converted into $\mathrm{CO}_{2}$ with energy. While in anaerobic conditions, via the glycolysis, combining lactate dehydrogenase, glucose is converted into lactate with energy. With receptor-ligand interaction, the cellular physiological activities will be affected.
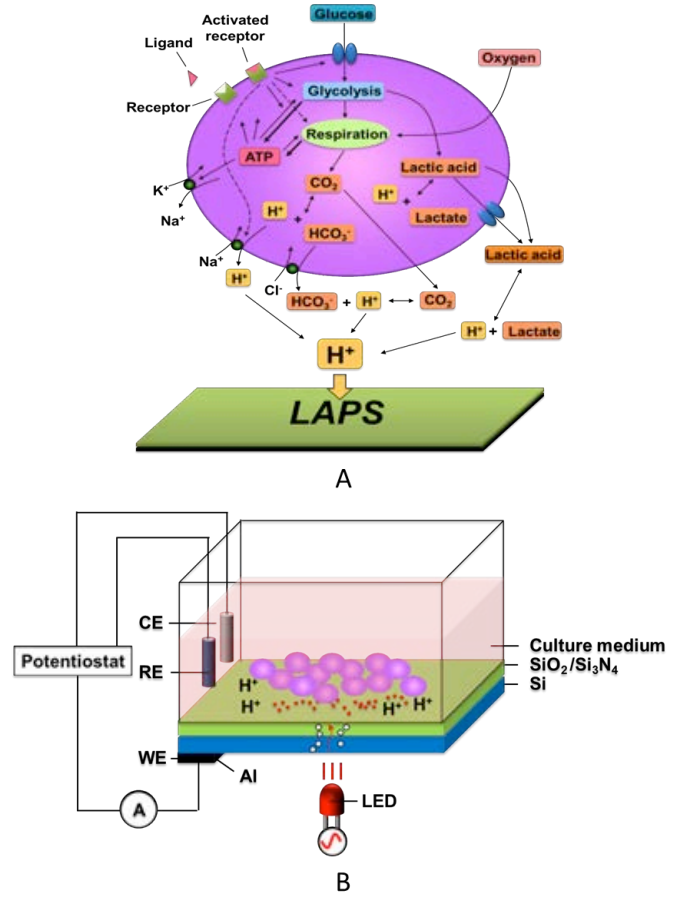

Fig.1. (A) Schematic of various cellular metabolism and processes induced extracellular acidification (B) $\mathrm{H}^{+}$concentration detection principle by LAPS. 
The corresponding ATP consumption is compensated by the increased uptake and metabolism of glucose, which results in increase of acidic products. The extracellular acidification induced $\mathrm{H}^{+}$concentration can be detected by LAPS in the real time.[1] The lightaddressable potentiometric sensor (LAPS) is essentially a sensitive $\mathrm{pH}$-meter with an electrolyte/insulator/semiconductor

structure [2, 3]. (See Fig.1(B)) The insulator consists of $\mathrm{SiO}_{2}$ and $\mathrm{Si}_{3} \mathrm{~N}_{4}$, which can effectively separate the silicon substrate from the electrolyte. The insulating layer interacts with $\mathrm{H}^{+}$in the solution to form a group of silanol (Si-OH) and silamine (Si-NH2). The $\mathrm{H}^{+}$ concentration in the solution can affect the sensor surface potential [4]. If a bias voltage is applied onto the reference electrode of sensor, a depletion layer forms at the silicon-insulator interface. By illuminating the frequencymodulated infrared light at the backside of the sensor, a photocurrent with same frequency is generated. This ac photocurrent corresponds to a hole-electron pair creation by radiation absorption from the LED. The amplitude of photocurrent is a function of surface potential and can be detected from backside aluminum layer by the instrument $[5,6]$.

In this study, a high sensitivity biosensor based on LAPS was established for monitoring the cellular metabolism. Constant voltage detection mode was introduced to replace the conventional constant current mode. The sensitivity of LAPS surface potential was improved by reducing the thickness of the semiconductor substrate. The performance of biosensor will be determined by the basic characteristic experiments and the cellular experiments.

\section{Constant Voltage Detection}

Current-Voltage characteristic curve plays an important role in indicating the $\mathrm{pH}$ sensitivity of LAPS. A series of the sigmoid curve is similar with a $\mathrm{pH}$ spectrum which reflects the relationship between the induced photocurrent and the bias voltage applied to sensor LAPS under the different $\mathrm{pH}$. The sigmoid curves have three regions as shown in Fig. 2. In cutoff region, there is no photocurrent. In saturation region, the photocurrent is close to maximum. In working region, the photocurrent increases as bias voltage decreases (n-type LAPS). Conventionally, the method of LAPS $\mathrm{pH}$ detection was by constant current mode, which monitors the change of inflection point voltage $(\triangle X)$. And $C-V$ curve shifts toward positive potentials as the solution $\mathrm{pH}$ increases. The conversion of $\mathrm{pH}$ and inflection point voltage can be determined by calibration. The constant current mode was commonly used to detect the $\mathrm{H}^{+}$concentration corresponding to the sensitivity of Nernst equation directly [7, 8]. However, the photocurrent amplification of LAPS was ignored. If the LAPS has the large amplification factor of $k(n A / m V)$ in the linear working region, the constant voltage mode will be more suitable than constant current mode to detect the $\mathrm{H}^{+}$concentration in the range of normal extracellular $\mathrm{pH}$.

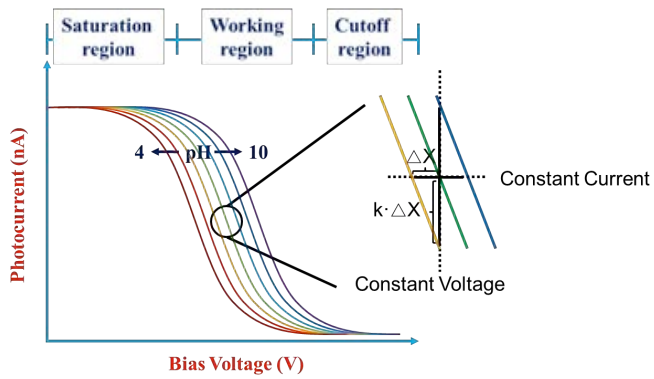

Fig.2. Typical C-V curves of LAPS sensor under the different $\mathrm{pH}$ and the monitoring parameters under constant current mode and the constant voltage mode.

\section{Sensor Fabrication}

LAPS device usually consists of the heterostructure of $\mathrm{Si} / \mathrm{SiO}_{2} / \mathrm{Si}_{3} \mathrm{~N}_{4}$. N-type silicon wafers (500 um thick and 4 inches in diameter) were selected for LAPS chip with specific resistance of $10 \Omega \cdot \mathrm{cm}$ and orientation index of crystal orientation $\langle 100\rangle$. As illustrated in Fig.3, The silicon chip surface was insulated with a layer of $50 \mathrm{~nm} \mathrm{SiO}_{2}$ by thermally oxidation at $1000{ }^{\circ} \mathrm{C}$. Then a thin layer of $50 \mathrm{~nm}$ $\mathrm{Si}_{3} \mathrm{~N}_{4}$ was deposited by LPCVD as a sensitive and protective layer on the upper side of $\mathrm{SiO}_{2}$. Significantly, the bulk silicon was grinded to 100 um to increase the sensitivity in our chip design. An aluminum membrane, about $300 \mathrm{~nm}$ in thickness, was finally evaporated on the backside of the silicon chip to form an ohm contact.

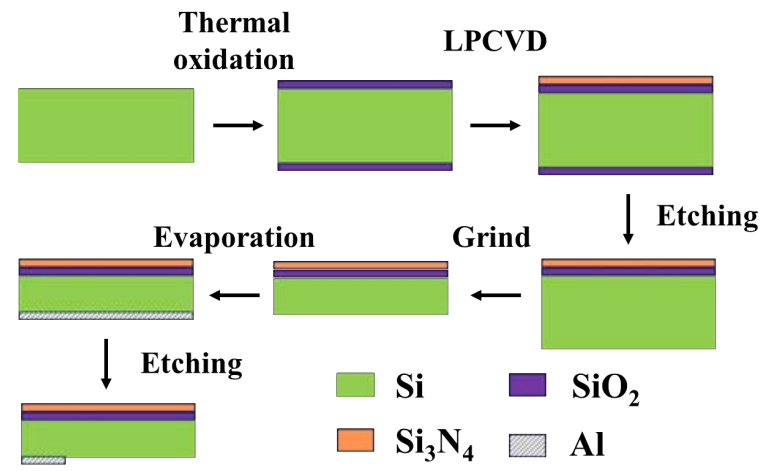

Fig.3. Fabrication processes of the improved LAPS. 


\section{LAPS performance experiment}

Before the extracellular acidification experiments, the performance of sensors should be determined by $\mathrm{C}-\mathrm{V}$ curve scanning and the sensor calibration. C-V curve (Fig.4) showed that the $100 \mathrm{um}$ thick LAPS had a significant large amplification factor $k$ than that of 500 um thick LAPS.

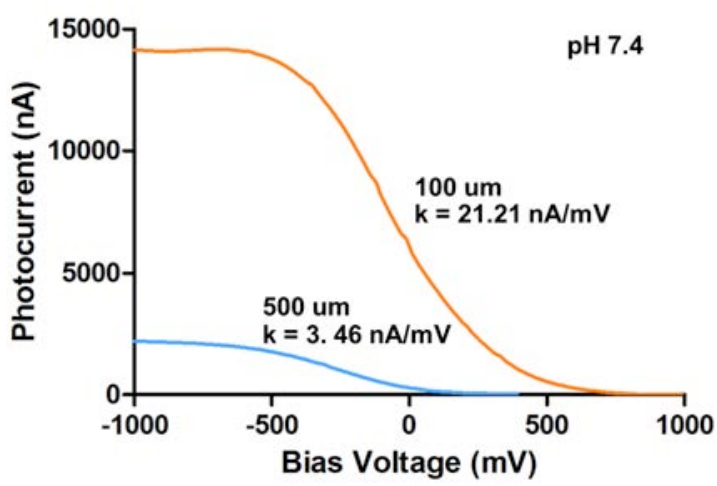

Fig.4.C-V curve of 100 um and 500 um thick LAPS at the $\mathrm{pH} 7.4$.
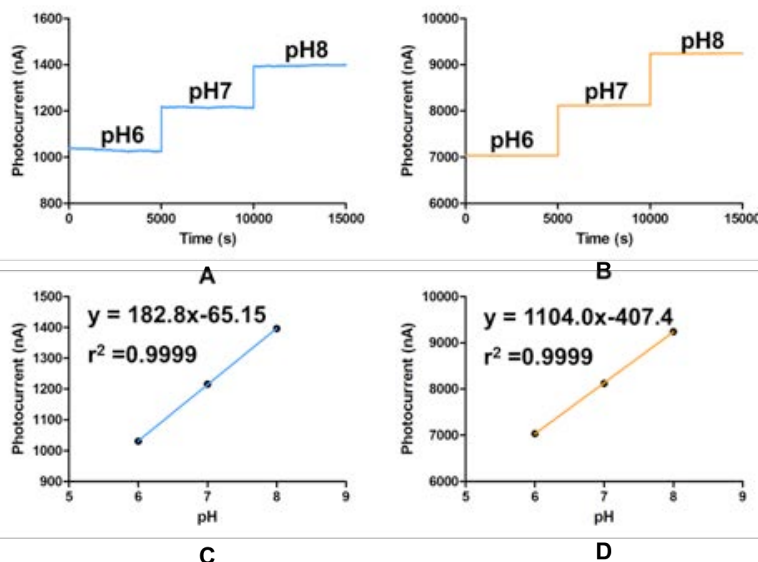

Fig.5. Calibration of 100 um and 500 um LAPS from $\mathrm{pH} 6$ to $\mathrm{pH} 8$ under the constant voltage detection mode.

After C-V curve scanning, the sensor chip was calibrated from $\mathrm{pH} 6$ to $\mathrm{pH} 8$. Each $\mathrm{pH}$ was recorded for about $5000 \mathrm{~s}$ to calculate the mean of its raw data Fig.5(A) and (B). From the calibration curve in the Fig.5(C) and (D), the sensitivity of improved LAPS was about 1104 $\mathrm{nA} / \mathrm{mV}$ versus $182.8 \mathrm{nA} / \mathrm{mV}$ of original LAPS. The calibration results also verified that the 100 um LAPS has a high sensitivity in the considered range, which can satisfy the requirements of cellular metabolic experiments.

\section{Human breast cell line culture}

The MCF-7 culture results were displayed in the Fig. 6. The $50 \mathrm{k} / \mathrm{ml}$ Cells were dense in 48 hours later (See Fig. 6(A)). After the DiffQuik dye, we can conclude that the MCF-7 developed well with the normal state in 48 hours culture, and the $50 \mathrm{k} / \mathrm{ml} \mathrm{MCF-7} \mathrm{closed} \mathrm{to} \mathrm{the} 95 \%$ fusion level in the sensor (Fig. 6(B)) which satisfied the detection requirement.

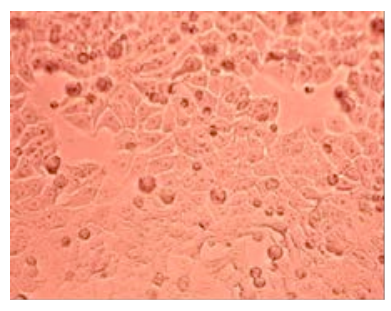

A

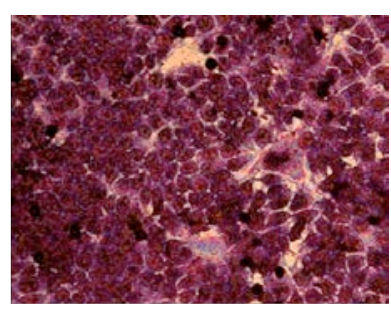

B
Fig.6. Human breast cancer cell MCF-7 cultured in 48 hours later $(A)$ and DiffQuik dye (B)

\section{MCF-7 Extracellular acidification experiment}

Finally, MCF-7 Extracellular acidification experiments were carried out to test the performance of the improve LAPS and constant detection mode. The metabolic activities of MCF-7 cells were monitored by the secretion of the cellular acidic metabolites. From the results in Fig.7, we can see that the extracellular acidification can be detected in a relative short time in the experiment.

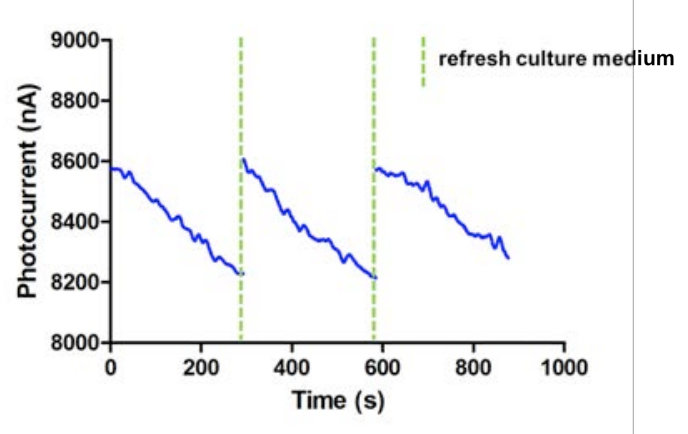

Fig.7.Typical result of extracellular acidification experiment based on MCF-7 cells.

\section{Conclusion}

We engaged in developing a high sensitivity biosensor by introducing the constant current detection mode and improving the structure of the light-addressable potentiometric sensor. By employing human breast cancer cell line MCF-7, the performance of high sensitivity biosensor was proved for detecting extracellular acidification rate.

\section{Acknowledgements}

This work was supported by the National Natural Science Foundation of China (Grant No. 81027003), the Zhejiang Provincial Science and Technology Major Special Program (No. 2010C14006). 


\section{References}

[1] X. M. Li, R. M. Schwartz, E. Y. Cesar, H. Y. Wang, An integrated microcomputer system using immobilized cellular electrodes for drug screening, Computers in biology and medicine 18(5):367376 (1988).

[2] D. G. Hafeman, J. W. Parce, H. M. McConnell, Light-addressable potentiometric sensor for biochemical systems, Science 240(4856):11821185 (1988).

[3] J. C. Owicki, L. J. Bousse, D. G. Hafeman, G. L. Kirk, J. D. Olson, H. G. Wada, et al., The lightaddressable potentiometric sensor: principles and biological applications, Annu Rev Biophys Biomol Struct 23(1):87-114 (1994).

[4] L. Bousse, N. F. De Rooij, P. Bergveld, The influence of counter-ion adsorption on the $\psi_{0} / \mathrm{pH}$ characteristics of insulator surfaces, Surface science 135(1):479-496 (1983).

[5] F. Hafner, Cytosensor ${ }^{\circledR}$ Microphysiometer: technology and recent applications, Biosensors and Bioelectronics 15(3):149-158 (2000).

[6] H. McConnell, J. Owicki, J. Parce, D. Miller, G. T. Baxter, H. Wada, et al., The cytosensor microphysiometer: biological applications of silicon technology, Science 257(5078):1906-1912 (1992).

[7] T. Wagner, C. Rao, J. Kloock, T. Yoshinobu, R. Otto, M. Keusgen, et al., "LAPS Card"-A novel chip card-based light-addressable potentiometric sensor (LAPS), Sensors and Actuators B: Chemical 118(1):33-40 (2006).

[8] T. Wagner, R. Molina, T. Yoshinobu, J. P. Kloock, M. Biselli, M. Canzoneri, et al., Handheld multichannel LAPS device as a transducer platform for possible biological and chemical multi-sensor applications, Electrochimica Acta 53(2):305-311 (2007). 